\title{
Prevalence of gestational thrombocytopenia among selected group of pregnant women attended to soba university hospital, Sudan, July-September 2016
}

\begin{abstract}
Background: Gestational thrombocytopenia, also known as incidental thrombocytopenia of pregnancy, is the commonest cause of thrombocytopenia in pregnancy occurring in approximately $75 \%$ of cases, it occurs in the middle of the second trimester and the third trimester and is not associated with maternal bleeding.
\end{abstract}

Rationale: Gestational thrombocytopenia may lead to severe complications for both pregnant lady and her fetus.

Objectives: To know the prevalence of gestational thrombocytopenia among selected group of pregnant ladies.

Materials and methods

Descriptive cross sectional study, blood specimen was collected from 100 pregnant ladies and examined by full automated haematological analyzer.

Result: There is no reported case of gestational thrombocytopenia among all pregnant ladies involved in the study $(0 \%)$.

Discussion: Globally gestational thrombocytopenia is considered when platelets count is below 116.000cell/ $\mu$ l of blood, no one of participants down to this number then the percentage of gestational thrombocytopenia among the study group $(0 \%)$ is lower than the normal reference range of gestational thrombocytopenia (7-8\%). Conclusion: There is no gestational thrombocytopenia among the pregnant ladies involved in the study.

Recommendation: Further studies should be done involving pregnant ladies from different racial group to know the effect of race on the number of platelets during pregnancy.

\author{
Volume 7 Issue 6 - 2018
}

\author{
Mosab Nouraldein Mohammed Hamad,' \\ Dakeen Khalifa Idam, ${ }^{2}$ Hana Alhag Alshazali ${ }^{3}$ \\ 'Medical Laboratory department, Faculty of Health Science, \\ Elsheikh Abdallah Elbadri University, Sudan \\ ${ }^{2}$ Pathology department, Soba university hospital, Sudan \\ ${ }^{3}$ Microbilogy department, Alzaeim Alazhari University, Sudan
}

Correspondence: Medical Laboratory department, Faculty of Health Science, Elsheikh Abdallah Elbadri University, Sudan, Email musab.noor13@gmail.com

Received: January 04, 2018 | Published: December 26, 2018

Keywords: gestational thrombocytopenia, pregnant women

\section{Introduction}

Thrombocytopenia is a condition in which you have a low blood platelet count. Platelets (thrombocytes) are colorless blood cells that help blood clot. Platelets stop bleeding by clumping and forming plugs in blood vessel injuries. Thrombocytopenia often occurs as a result of a separate disorder, such as leukemia or an immune system problem. Or it can be a side effect of taking certain medications. It affects both children and adults.

Thrombocytopenia, or a low blood platelet count, is encountered in $7-12 \%$ of all pregnancies. Women are more commonly diagnosed with platelet disorders during pregnancy since screening is done as part of the initial clinic evaluation with automated blood counts. Thrombocytopenia can result from a wide range of conditions with several of them being pregnancy related. Platelets are nonnucleated cells derived from megakaryocytes in the bone marrow and normally live in the peripheral circulation for as long as 10 days. Platelets play a critical initiating role in the hemostatic system.

The normal range of platelets in nonpregnant women is 150,000 $400,000 / \mu \mathrm{L}$ Average platelet count in pregnancy is decreased $(213,000 / \mu \mathrm{L}$ vs $250,000 / \mu \mathrm{L})$, and declines as pregnancy progresses. Change in platelet count is due to hemodilution, increased platelet consumption, and increased platelet aggregation driven by increased levels of thromboxane A2. Thrombocytopenia can be defined as platelet count less than $150,000 / \mu \mathrm{L}$ or platelet count below the 2.5 th percentile for pregnant patients $(116,000 / \mu \mathrm{L})$.

Classification of thrombocytopenia in pregnancy is arbitrary and not necessarily clinically relevant. Mild thrombocytopenia is $100,000-$ $150,000 / \mu \mathrm{L}$. Moderate thrombocytopenia is $50,000-100,000 / \mu \mathrm{L}$. Severe thrombocytopenia is $<50,000 / \mu \mathrm{L}$. In normal pregnancies, $7.6 \%$ of women present with mild thrombocytopenia during pregnancy, and $65 \%$ of them will not be associated with any pathology. Any pregnant patient with a platelet count of less than $100,000 / \mu \mathrm{L}$ should undergo further clinical and laboratory assessment. ${ }^{2}$

Although women with normal pregnancy experience a low platelet count, women experiencing a continuous drop in platelet will be diagnosed with thrombocytopenia and women with levels greater than $70,000 / \mu \mathrm{L}$ will be diagnosed with gestational thrombocytopenia. ${ }^{3}$ although the pathophysiology of gestational thrombocytopenia is not known, it is known to be associated with two factors:

a) Increased platelet activation, which is suspected to occur at placental circulation.

b) Increased peripheral consumption of platelets because of their reduced lifespan in pregnancy. ${ }^{4}$

Evaluation and management of thrombocytopenia during pregnancy and postpartum may be challenging because there are 
many potential causes, some directly related to the pregnancy and some unrelated. For many of the causes there are no diagnostic laboratory tests. Management options may have the potential for serious complications for both mother and fetus and may require urgent decisions about delivery, and there may be concerns about fetal thrombocytopenia. ${ }^{5}$ Hypertensive disorders account for approximately $20 \%$ and immune thrombocytopenic purpura for about $3-4 \%$ of gestational thrombocytopenia.

Immune thrombocytopenic purpura: A more rare cause of thrombocytopenia in pregnancy is the immune thrombocytopenic purpura (ITP), an autoimmune disorder characterized by the antiplatelet glycoprotein antibodies that stimulate the platelet destruction in the spleen. There are two types of ITP: the acute form that is common in children, is associated with viral infections and is selflimiting and the chronic form which predominantly affects women. The diagnosis is clinical. Thrombocytopenia in ITP is generally moderate but with platelet count usually below 100.000/iL and the symptoms are in direct relation to the platelet levels. Patients could be completely asymptomatic or present ecchymosis, petechiae, purpura, gums bleeding or menorrhagia. Unlike gestational thrombocytopenia, ITP can occur anytime during pregnancy. Moreover, most pregnant women with ITP may have a history of thrombocytopenia prior to pregnancy or may present with other immune mediated diseases. The platelet count does not spontaneously improve postpartum and the therapeutic response to steroids or IVIG (intravenous immunoglobulins) contributes to the diagnosis of ITP.

Pregnancy does not worsen the outcome of ITP, but there may be adverse fetal and maternal consequences in some cases. Although rare, spontaneous bleeding is the main maternal risk especially when the platelet count falls below 20.000/iL. Steroids or IVIG are recommended before 36 weeks if platelet count is under $30.000 / \mathrm{iL}$, the patient is symptomatic or an invasive procedure is considered. Around delivery, the aim is to maintain platelet count above 50.000/ $i \mathrm{~L}$, the level considered safe for both vaginal and cesarean delivery. Intravenous immunoglobulin at a dose of $1 \mathrm{~g} / \mathrm{kg}$ has a relatively rapid therapeutic response (within 1-3 days) or prednisone $1 \mathrm{mg}$ / $\mathrm{kg}$ with a therapeutic response within 2-14 days could be used for treatment during pregnancy. The first-line therapy drug, prednisone, is considered safe, but can induce or exacerbate gestational diabetes, maternal hypertension, osteoporosis, weight gain and psychosis. Prednisone is metabolized by placenta but high doses have been linked to fetal adrenal suppression and a small increase in incidence of cleft lip and palate if used in the first trimester. In severe cases of thrombocytopenia, unresponsive to alternative therapies, splenectomy can safely be performed, especially in second trimester of pregnancy. Pre-splenectomy immunizations are safe in pregnancy, as all the vaccines required are inactivated. Platelet transfusion is not indicated for ITP treatment due to the consumptive mechanism of this condition. When emergency cesarean delivery is required with a platelet count under $50.000 / \mathrm{iL}$, platelet transfusions in association with IVIG is recommended. Immune thrombocytopenic purpura is not an indication for caesarean delivery.

The IgG anti-glycoprotein platelet antibodies can cross the placenta and could induce neonatal thrombocytopenia (with an estimated risk of 5-10\%). ${ }^{4}$ There is no correlation between maternal and fetal platelet levels and maternal response to treatment does not protect the fetus from a possible neonatal thrombocytopenia. Neonatal platelet levels should be determined at birth and further daily monitored. The lowest levels are recorded during days 2 to 5 postnatally. When platelet count in the newborn is below $50.000 / \mathrm{iL}$, there is a risk of $0.5-1.5 \%$, of intracranial hemorrhage and a head ultrasound is recommended even in the absence of symptoms. When the platelet count is between 30.000 to $50.000 /$ iL, IVIG treatment should be started. With platelet count under $30.000 / \mathrm{iL}$ platelet transfusion along with IVIG is recommended.

\section{Thrombocytopenia associated with hypertensive disorders:} Thrombocytopenia associated with hypertensive disorders (preeclampsia, eclampsia, HELLP syndrome, acute fatty liver of pregnancy) is the second leading cause of thrombocytopenia in pregnancy. Thrombocytopenia occurring in this context is a sign of hypertensive disorder severity. Levels rarely fall below $20.000 / \mu \mathrm{L}$. Preeclampsia causes about $20 \%$ of cases of thrombocytopenia in pregnancy. Thrombocytopenia is sometimes the only initial sign of this condition, predating all the other laboratory changes. HELLP syndrome (hemolysis, elevated liver function tests, and low platelets) is another pregnancy specific disorder and it complicates $10-20 \%$ of cases of severe preeclampsia. The syndrome can occur without proteinuria ( $25 \%$ of cases) or hypertension ( $40 \%$ of cases) and the diagnosis may then be missed. About $70 \%$ of the cases develop before delivery, the majority between the 27th and 37th gestational week, but in some women the signs suggestive of HELLP syndrome may occur postpartum ( $30 \%$ of cases). A platelet count of $<100.000 / \mu \mathrm{L}$ is one of the diagnostic criteria of HELLP syndrome.

The pathophysiology is similar to that of pre-eclampsia, with endothelial damage and release of tissue factors and coagulation activation. Acute fatty liver of pregnancy (AFLP) is a rare (1:7000 - 1:20000 pregnancies), but severe condition of the third trimester of pregnancy. Clinical manifestations such as abdominal pain, nausea, vomiting, anorexia, in conjunction with several specific laboratory changes such as severe hypoglycemia, hyperuricemia, markedly elevated transaminases, renal impairment with elevated creatinine, blood pressures in the normal range, may lead to the diagnosis of acute fatty liver of pregnancy. Thrombocytopenia associated with this pathology is sometimes severe, with a platelet count under 20.000/ $\mu \mathrm{L}$. The only effective treatment of preeclampsia/ eclampsia, HELLP Syndrome and acute fatty liver of pregnancy is delivery. The aim is to maintain platelet count around the safety limit of above 50.000/ iL. Dexamethasone $10 \mathrm{mg}$ every 12 hours, 2 to 4 doses antepartum and two doses postpartum is usually recommended. If hemolysis, thrombocytopenia and renal dysfunction worsen plasmapheresis could be used. In the absence of other complications such as disseminated intravascular coagulation (DIC) or renal dysfunction, the platelet count usually returns to normal values by day 4 postpartum and reach $100.000 / \mu \mathrm{L}$ by day 6 .

The pathophysiologic mechanism of thrombocytopenia in hypertensive disorders is the thrombotic microangiopathy characterized by endothelial injury, followed by platelet aggregation and thrombus formation in small vessels. The mark of thrombotic microangiopathy are the presence of schistocytes on peripheral blood smear and increased bilirubin $>1.2 \mathrm{mg} / \mathrm{dL}$, decreased haptoglobin $<25$ $\mathrm{mg} / \mathrm{dl}$ and increased LDH biochemically.

Thrombotic microangiopathy is the pathophysiological mechanism for thrombotic thrombocytopenic purpura (TTP) and the hemolytic uremic syndrome (HUS), two pathologic conditions difficult to distinguish one from another and from preeclampsia/ HELLP syndrome and AFLP. Both TPT and HUS are not pregnancy 
specific pathologic conditions, occurring with higher incidence (1: 25000) especially towards the end of the pregnancy.

TTP is defined by a pentad of microangiopathic hemolytic anemia, thrombocytopenia, fever, neurological manifestations, and renal impairment. Creatinine levels in TTP are not increased and the median platelet count is typically $10-30000 / \mu \mathrm{L}$. However, TTP can present without the full pentad, up to $35 \%$ of patients not having neurological signs at presentation and renal abnormalities and fever are not prominent features. Neurological presentation could include a myriad of signs as headache, altered personality, reduced cognition, transient ischemic attacks, different levels of consciousness alteration including coma.

Pregnancy can be the trigger event for approximately $5-25 \%$ of TTP cases. TTP occurs in the second part of pregnancy and sometimes in postpartum, while it remains unusual during the first trimester of gestation. If TTP develops in the first trimester, regular plasma exchange may allow continuation of pregnancy. There are two types of TTP: a familial type characterized by a constitutional deficiency of ADAMTS 13 (the Upshaw-Schulman Syndrome USS) and a nonfamilial type characterized by the production of anti- ADAMTS 13 antibodies (acquired). ADAMTS 13 is a metalloprotease acting to cleave the von Willebrand factor multimers and thus it prevents thrombus formation by the impaired platelet aggregation. A deficiency in ADAMTS 13 activity can be seen also in DIC, HUS, preeclampsia and the HELLP syndrome $(<40 \%$ but $>10 \%)$, but the severe deficiency $<5 \%$ of the normal activity, is specific for TTP. Severely reduced ADAMTS 13 activity or the presence of an inhibitor or IgG antibodies confirm the diagnosis of TTP. Blood must be taken prior to treatment to asses' baseline ADAMTS 13 activity. Globally, the stillbirth rate in pregnancy-associated TTP is reported to be about $40 \%$, mainly due to intrauterine fetal death (IUFD), spontaneous abortions and prematurity. The major pathophysiological mechanism for intrauterine fetal growth restriction and death may be placental ischemia. No cases of fetal thrombocytopenia or hemolytic anemia have been described in maternal TTP, although the anti ADAMTS 13 IgG cross the placental barrier. Because of the risk of IUFD caused by the thrombosis of the decidua arterioles and placental hypoperfusion, it is recommended to perform a careful fetal monitoring by Doppler ultrasound of the uterine arteries and fetal arteries to detect signs of fetal distress or intrauterine growth restriction. Delivery may be required earlier and fetal prognosis is dependent on the gestational age at birth.

HUS has a common mechanism with TTP, but the distinctive feature is the more severe kidney impairment with HUS. There are two types of HUS: the typical form, represents $90 \%$ of cases of HUS, is common in children and is caused by E. coli $\mathrm{O} 157: \mathrm{H} 7$ which produces the Shiga-toxin and the atypical form which is usually associated with pregnancy and is related to a congenital defect of the alternative pathway of the complement system. It occurs in most cases postpartum. Both TTP and HUS can have immediate fetal and maternal consequences in the absence of treatment, so establishing an early diagnosis is very important. Therefore, every pregnant woman with thrombocytopenia and microangiopathic hemolytic anemia should be considered suspicious for TTP or HUS until proven otherwise. The laboratory investigations are useful in establishing the diagnosis are microangiopathic hemolytic anemia, presence of schistocytes in blood smear, normal coagulation tests and increased creatinine as a marker of renal injury. Unlike preeclampsia/HELLP syndrome, delivery does not influence the outcome of the disease; the first-line treatment in cases of TTP/ HUS is plasmapheresis and administration of fresh frozen plasma as soon as possible. Plasmapheresis is repeated until the normal levels of platelets and LDH are reached. For kidney impairment temporary dialysis could be required.

For atypical HUS, eculizumab, a monoclonal anti-C5 inhibitor appears a promising agent. Eculizumab was approved for treatment of complement mediated HUS by Food and Drug Administration and the European Medicine Agency. Eculizumab has been proven efficient in paroxysmal nocturnal hemoglobinuria during pregnancy and treatment with eculizumab is generally safe. The main concerns are the high costs and a small risk of Neisseria infection, requiring vaccination prior to treatment. The risk of recurrence in subsequent pregnancies in women with acquired TTP is about $20 \%$. Regular plasma exchange and serial monitoring of ADAMTS 13 activity have a benefit for both the maternal and the baby outcome. In congenital TTP the risk for relapse in the subsequent pregnancies is $100 \%$ in the absence of prophylaxis. Plasma exchange should be initiated at the end of first trimester and repeated throughout the pregnancy and postpartum if necessary.

Pseudothrombocytopenia is often seen when anticoagulants such as EDTA are used when collecting blood. They induce platelet aggregation and therefore false low platelet count. A peripheral blood smear is important for establishing the diagnosis (the platelets are seen arranged in stacks). Disseminated intravascular coagulation (DIC) is characterized by the activation of the coagulation system, microvascular thrombus formation in different organs and multiple organ failure. Biochemically is defined by an increase of the PT, aPTT, thrombocytopenia, decreased fibrinogen, fibrin degradation product accumulation and the presence of D-dimers. In pregnancy, DIC is caused by a number of causes, the most important being placental abruption, amniotic embolus and uterine rupture. Von Willebrand disease type IIb is a rare cause of thrombocytopenia in pregnancy. There is a qualitative defect of the von Willebrand factor inducing high affinity to the platelet receptor of glycoprotein $1 \mathrm{~b}$. Thrombocytopenia is explained by increased aggregation. Women with this condition may present with thrombocytopenia for the first time in pregnancy and platelets can drop to values below 20.000-30.000/iL. Levels of von Willebrand factor and factor VIII should be increased to $>50$ $\mathrm{IU} / \mathrm{dL}$ to cover any surgical procedures. Platelet transfusions may be required together with the factor VIII replacement if the platelet count is $<20.000 / \mathrm{iL}$ antenatally, prior to any invasive procedures, if bleeding occurs or if platelet count is $<50.000 / \mathrm{iL}$ around delivery. ${ }^{6}$ General measures, including avoidance of aspirin, non-steroidal antiinflammatory drugs, and intramuscular injections, should also be considered depending on the platelet level and stability. ${ }^{7}$

Rationale: Gestational thrombocytopenia may lead to severe complications for both pregnant lady and her fetus.

Objectives: To know the prevalence of gestational thrombocytopenia among selected group of pregnant ladies.

\section{Material and methods}

Study design: Descriptive cross sectional study

Study area: Soba university hospital, Khartoum, Sudan

Study population: Pregnant Ladies attended to Soba university hospital 
Study period: July to September 2016

Sample size: 100 pregnant ladies

Specimens: Venous blood specimen was collected from each participant and preserved in EDTA anticoagulant.

Specimen's laboratory analysis: All specimens were analyzed by full automated haematological analyzer (sysmex haematological analyzer).

Ethical Consideration: All participants were consent to involved in the study.

\section{Results}

There is no reported case of gestational thrombocytopenia among all pregnant ladies involved in the study $(0 \%)$.

\section{Discussion}

Globally gestational thrombocytopenia is considered when platelets count is below $116.000 \mathrm{cell} / \mu \mathrm{l}$ of blood, no one of participants down to this number then the percentage of gestational thrombocytopenia among the study group ( $0 \%)$ is lower than the normal reference range of gestational thrombocytopenia (7-8\%).

\section{Conclusion}

There is no gestational thrombocytopenia among the pregnant ladies involved in the study.

\section{Recommendations}

Further studies should be done involving pregnant ladies from different racial group to know the effect of race on the number of platelets during pregnancy.

\section{Acknowledgments}

None.

\section{Conflicts of interest}

The author declares there are no conflicts of interest.

\section{References}

1. Thrombocytopenia (low platelet count).

2. Thrombocytopenia in Pregnancy. Obstetrics \& Gynecology. 2016.

3. https://en.wikipedia.org/wiki/Gestational_thrombocytopenia.

4. Thrombocytopenia (Low Platelet Count) During Pregnancy: Causes, Symptoms, \& Treatment. Safety. 2018.

5. James N George, Jennifer J Mcintosh. Thrombocytopenia in pregnancy. 2018.

6. Ciobanu AM, Colibaba S, Cimpoca B, et al. Thrombocytopenia in pregnancy. Maedica (Buchar). 2016;11(1):55-60

7. Bethan Myers. Diagnosis and management of maternal thrombocytopenia in pregnancy. BJH. 2012;158(1):3-15 